\section{Association of outer retinal layer morphology with visual acuity in patients with retinal vein occlusion: SCORE Study Report 13}

\author{
Abstract \\ Purpose To assess associations between \\ visual acuity (VA) and the status of the \\ photoreceptor inner segment-outer segment \\ (IS-OS) junction in a subset of patients in \\ the Standard Care vs COrticosteroid for \\ REtinal Vein Occlusion (SCORE) Study. \\ Methodology High-resolution time domain \\ optical coherence tomography (OCT) scans of \\ study eyes from a single site participating in \\ the SCORE Study were evaluated. Integrity \\ of the IS-OS junction in the central subfield \\ was evaluated using a three-step scale: \\ absent, abnormal or normal. Associations of \\ the IS-OS status with ETDRS VA letter score \\ and center point thickness (CPT) were \\ investigated. \\ Results Baseline OCTs of 42 eyes were \\ evaluated. The IS-OS junction was absent in \\ $30(71 \%)$ and abnormal in $12(29 \%)$. At month \\ 12, the IS-OS junction was absent in $\mathbf{1 8}$ \\ (43\%), abnormal in $12(28 \%)$, and normal in \\ $12(28 \%)$ eyes. At baseline, IS-OS status was \\ significantly associated with CPT, but not \\ with VA. At month 12, IS-OS status was \\ significantly associated with CPT and VA, \\ that is, absent or abnormal IS-OS was \\ associated with increased CPT and worse VA. \\ Change in IS-OS status was not associated \\ with change in CPT $(P=0.8)$. Worsening of \\ IS-OS status was associated with loss of VA \\ and improvement in IS-OS status to normal \\ was associated with gain in VA $(P=0.03)$. \\ Conclusion In this data set with long-term \\ follow-up of OCTs as part of the SCORE \\ Study, there is a correlation between change \\ in IS-OS status and VA. This supports \\ further evaluation of outer retinal \\ morphology in larger data sets.
}

A Domalpally', Q Peng ${ }^{1}$, R Danis ${ }^{1}$, B Blodi ${ }^{1}$, IU Scott ${ }^{2}, M$ Ip ${ }^{1}$ and the SCORE Study Research Group
Eye (2012) 26, 919-924; doi:10.1038/eye.2012.59; published online 27 April 2012

Keywords: photoreceptor layer; inner segmentouter segment junction; outer retina; retinal vein occlusion; SCORE Study

\section{Introduction}

Retinal thickness parameters as assessed by optical coherence tomography (OCT) have been used extensively for clinical trial eligibility, clinical trial endpoints, and clinical management of retinal diseases. ${ }^{1-5}$ However, the correlation between OCT-measured central retinal thickness and visual acuity (VA) is only modest among a variety of retinal diseases. ${ }^{6-10}$ Emerging literature suggests that both qualitative and quantitative features of the outer retina may correlate with VA in various macular pathologies, including retinal vein occlusion (RVO). ${ }^{9,11,12-14}$ If so, outer retinal morphology may be an important predictor of visual function and emerge as an important endpoint in clinical trials of RVO and other conditions.

Spectral domain OCT (SD OCT) technology has led to progress in outer retinal layer segmentation to enable specific layer measurements, such as photoreceptor length and outer segment length, although the algorithms have been thus far applied to eyes with no known disease or a few selected pathologies. ${ }^{15-20}$ Controversy still exists in the identification of precise outer retinal layers. ${ }^{18,21}$ The disruption of retinal architecture in pathology poses a challenge to the segmentation algorithm, which could result in erroneous
${ }^{1}$ Fundus Photograph Reading Center, University of Wisconsin, Madison, WI, USA

${ }^{2}$ Departments of Ophthalmology and Public Health Sciences, Penn State College of Medicine, Hershey, PA, USA

Correspondence: RP Danis, Fundus Photograph Reading Center, 8010 Exclesior Drive, Suite 100, Madison, WI 53717, USA

Tel: + 16084100612 ;

Fax: + 16084100568 .

E-mail: rdanis@rc.ophth. wisc.edu

Received: 14 December 2011

Accepted in revised form: 29 January 2012

Published online: 27 April 2012

Data have been presented at Association for Research in Vision and Ophthalmology (ARVO) 2010. 
thickness measurements. ${ }^{22}$ Integrity of the photoreceptor inner segment-outer segment (IS-OS) junction has been shown to correlate well with VA in cross sectional studies of patients with diabetic macular oedema, RVOs, and neovascular age-related macular degeneration. ${ }^{12-14,23,24}$ Longitudinal evaluation in large data sets would help confirm the validity of this variable.

The Standard Care vs COrticosteroid for REtinal Vein Occlusion (SCORE) Study employed high resolution time domain OCT (TD OCT; Stratus, Carl Zeiss Meditec, Dublin, CA, USA) images of eyes with macular oedema associated with RVO. The SCORE Study results showed only a moderate correlation between change in VA and change in OCT-measured center point thickness (CPT) ${ }^{25,26}$ The purpose of this SCORE ancillary study is to evaluate the correlation between the IS-OS status and concurrent VA, and also the relationship between change in IS-OS status and change in VA in a longitudinal set of high resolution TD OCT images of patients with RVO.

\section{Materials and methods}

The SCORE Study design and baseline characteristics of the SCORE Study participants have been published previously. ${ }^{1}$ OCT scans were obtained using the Stratus OCT3 machine by certified imaging technicians at baseline and every 4 months thereafter for 36 months. The scanning protocol included the fast macular thickness scan protocol (128 A scans/B scan) and the high resolution cross hair scan protocol (512 A scans/B scan). The SCORE Study reading centre utilized paper prints of the scans for evaluation. However, the evaluation protocol for outer retina required gray scale images for identifying the IS-OS junction and photoreceptor layer. To accommodate this, the OCT scans included in the current study were evaluated in the manufacturer's review software (Carl Zeiss Meditec). As the project involved retrieving digital OCT scans, evaluation was limited to scans from a single SCORE Study site only (University of Wisconsin). The high resolution cross hair scans were utilized as the resolution of fast macular scans was insufficient for evaluating the IS-OS junction.
Cross hair scans were viewed in the manufacturer's review software using the align scan function with images displayed in gray scale. Scan centration was assessed and the length of the B scan corresponding to the central subfield was identified. Using both the horizontal and vertical cross hairs, the IS-OS junction in the central subfield was evaluated using a three step scale: normal, abnormal, and absent (Figure 1). 'Normal' IS-OS junction indicated a well-delineated hyper reflective signal passing through the entire length of the central subfield. 'Abnormal' IS-OS junction was defined as disruption in the signal intensity so that it appeared speckled or irregular, yet still visible. An 'absent' IS-OS junction indicated that the layer was not visualized. The lack of visualization could be due to the absence owing to pathology or due to blocked signal (haemorrhage, large cyst) or change in orientation of photoreceptors (subretinal fluid) or poor signal quality. Change in IS-OS status from baseline to month 12 was categorized as improved, no change, or worsened. Improved IS-OS status was defined as a change in IS-OS status from absent to abnormal, absent to normal, or abnormal to normal. Worsening of IS-OS status indicated that eyes changed from abnormal IS-OS at baseline to absent IS-OS at month 12 .

The assessment was made separately for the two cross hair scans and the worse grade was selected for the eye. The reproducibility of the three step scale was assessed on a pilot data set evaluated by two independent graders (separate from the SCORE Study data set; $n=30$ eyes). Agreement for evaluation of IS-OS status was $80.6 \%$ ( $\kappa$ 0.71). All images thereafter were graded independently by a single evaluator. Each visit scan was graded independently without comparison with previous or baseline scans.

Photoreceptor length was measured as the distance between the outer plexiform layer (OPL) and the retinal pigment epithelium. This measurement was taken at three points in the central subfield of each cross hair scan using the software calipers, at the center point and $500 \mu \mathrm{m}$ on either side of the center point. Both the OPL and the retinal pigment epithelium had to be distinctly visible to position software calipers on at least one of the three points on a B scan without which the measurement
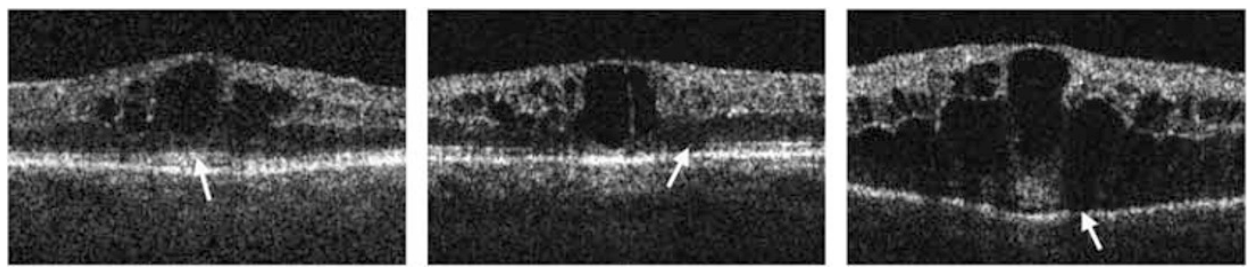

Figure 1 Evaluation of the photoreceptor inner segment-outer segment (IS-OS) status using the three step scale; left normal IS-OS, centre abnormal IS-OS and right absent IS-OS. 
was considered ungradable. All available measurements were used to obtain a mean photoreceptor length in the central subfield.

Measurement of the best corrected VA was performed using the electronic Early Treatment Diabetic Retinopathy Study VA method at $3 \mathrm{~m}$ by a SCORE Studycertified VA examiner.

Statistical analysis was performed using the KruskalWallis test to compare the distribution of VA and CPT across the three step scale (Wilcoxon rank-sum test when only two steps were available). As the data set was limited to a single site, analysis across treatment arms was not performed.

\section{Results}

OCT scans of 42 subjects enrolled at a single site were evaluated. At baseline, the IS-OS junction was absent in $30(71 \%)$ eyes, abnormal in $12(28 \%)$ eyes and normal in

Table 1 Baseline characteristics of eyes with absent and abnormal IS-OS status

\begin{tabular}{|c|c|c|}
\hline & $\begin{array}{l}\text { Absent } \\
(\mathrm{n}=30)\end{array}$ & $\begin{array}{l}\text { Abnormal } \\
(\mathrm{n}=12)\end{array}$ \\
\hline CRVO $n(\%)$ & $14(87.5 \%)$ & $2(12.5 \%)$ \\
\hline BRVO $n(\%)$ & $16(61.5 \%)$ & $10(38.5 \%)$ \\
\hline Subretinal fluid in central subfield $n(\%)$ & $8(26.7 \%)$ & $3(26.5 \%)$ \\
\hline Blocked signal in central subfield $n(\%)$ & $9(30.0 \%)$ & $2(16.7 \%)$ \\
\hline $\begin{array}{l}\text { Both subretinal fluid and blocked } \\
\text { signal in central subfield }\end{array}$ & $6(20.0 \%)$ & $1(8.3 \%)$ \\
\hline $\begin{array}{l}\text { Neither subretinal fluid nor blocked } \\
\text { signal }\end{array}$ & $7(23.3 \%)$ & $6(50.0 \%)$ \\
\hline
\end{tabular}

none. The 30 eyes with absent IS-OS junction were almost equally divided between central and branch RVO (Table 1). Retinal thickness at the centre point was greater and VA tended to be worse in these eyes than in those in which the IS-OS junction was classified as absent at baseline (Table 2). At month 12, eyes with absent IS-OS status had the worst VA, eyes with abnormal IS-OS status had intermediate VA, and eyes with normal IS-OS status had the best VA $(P<0.01)$. The trend in the distribution of VA letter score between the three categories was also seen at the month 4 and 8 follow-up visits.

Table 3 displays change in VA letter score for eyes with improved or no change in IS-OS status. There was a significant difference in the mean change in VA letter score between eyes with improved IS-OS and eyes with no change in IS-OS $(+6.0$ and -7.8 letters, respectively; $P=0.03$ ). There was no significant difference in the proportion of eyes with a gain in VA letter score of $\geq 15$ letters between the two categories, although there was a trend $(P=0.06)$. There was no difference in the distribution of mean change in CPT between the two categories $(P=0.8)$. A similar analysis at month 24 showed no significant difference in change in VA letter score with change in IS-OS status; eyes that demonstrated an improved IS-OS status had a mean VA gain of $1.69 \pm 26.9$ letters compared with a mean VA loss of $2.3 \pm 15.7$ letters in eyes that showed no change in IS-OS status $(P=0.29)$ (data not shown). In addition two eyes developed significant cataract at month 12 visits. The results did not change after excluding the two eyes.

Table 2 Comparison of IS-OS status at baseline and month 12 with other baseline parameters $(n=42)$

\begin{tabular}{|c|c|c|c|c|c|c|c|c|c|c|}
\hline & \multicolumn{3}{|c|}{ Absent } & \multicolumn{3}{|c|}{ Abnormal } & \multicolumn{3}{|c|}{ Normal } & \multirow[t]{2}{*}{$\mathrm{P}$ value } \\
\hline & $\mathrm{N}$ & Mean (SD) & Median & $\mathrm{N}$ & Mean (SD) & Median & $\mathrm{N}$ & Mean (SD) & Median & \\
\hline \multicolumn{11}{|l|}{ IS-OS status at baseline } \\
\hline VA letter score & 30 & $50.6(14.6)$ & 54.5 & 12 & $57.7(13.4)$ & 62.5 & 0 & - & - & 0.13 \\
\hline Centre point thickness $(\mu \mathrm{m})$ & 30 & $675.8(232.2)$ & 651.0 & 12 & $474.3(133.0)$ & 480.0 & 0 & - & - & $<0.01$ \\
\hline \multicolumn{11}{|l|}{ IS-OS status at month 12} \\
\hline VA letter score & 18 & $35.7(22.8)$ & 42.5 & 12 & $52.3(19.9)$ & 56.2 & 12 & $72.1(10.1)$ & 74.0 & $<0.01$ \\
\hline Centre point thickness $(\mu \mathrm{m})$ & 18 & $468.5(220.6)$ & 463.5 & 12 & $351.8(158.9)$ & 332.5 & 12 & $248.6(126.6)$ & 228.5 & 0.01 \\
\hline
\end{tabular}

Bold values are statistically significant.

Table 3 Change in IS-OS status between baseline and month 12 and change in visual acuity and center point thickness

\begin{tabular}{lccc}
\hline & \multicolumn{3}{c}{ IS-OS status change at M12 } \\
\cline { 2 - 4 } Change in VA at M12 & Improved $(\mathrm{N}=18)$ & No change $(\mathrm{N}=22)$ & P value \\
\hline Mean $(\mathrm{SD})$ change in VA & $+6.0(17.6)$ & $-7.8(20.0)$ & $0.03^{\mathrm{a}}$ \\
VA letter score $\geq 15$ letters & $7 / 18(39 \%)$ & $3 / 22(14 \%)$ & $0.06^{\mathrm{b}}$ \\
Mean change in center point thickness $(\mu \mathrm{m})$ & $-266.2(242.2)$ & $-242.3(259.5)$ & $0.80^{\mathrm{a}}$ \\
\hline
\end{tabular}

\footnotetext{
${ }^{\text {a }}$ Wilcoxon rank-sum test.

${ }^{\mathrm{b}}$ Test for comparison of proportion with $\geq 15$ letter improvement.
} 
Table 4 Change in retinal thickness at center point and change in visual acuity between baseline and month 12 by IS-OS status

\begin{tabular}{|c|c|c|c|c|}
\hline \multirow[t]{2}{*}{ IS-OS status at baseline } & \multicolumn{3}{|c|}{ IS-OS status at month 12} & \multirow[t]{2}{*}{$\mathrm{P}$ value } \\
\hline & Absent & Abnormal & Normal & \\
\hline \multicolumn{5}{|l|}{ Absent } \\
\hline$n$ & 16 & 6 & 8 & \\
\hline Change in mean CPT $\mu \mathrm{m}$ (SD) & $-277.1(279.7)$ & $-302.5(364.2)$ & $-322.1(158.0)$ & 0.73 \\
\hline Change in mean VA (SD) & $-10.3(22.5)$ & $-0.8(22.6)$ & $+8.9(15.4)$ & 0.11 \\
\hline \multicolumn{5}{|l|}{ Abnormal } \\
\hline$n$ & 2 & 6 & 4 & \\
\hline Change in mean $\mathrm{CPT} \mu \mathrm{m}$ (SD) & $-103.5(9.2)$ & $-149.5(184.2)$ & $-99.8(72.5)$ & 0.66 \\
\hline Change in mean VA (SD) & $-5.5(13.4)$ & $-1.3(10.0)$ & $+10.5(14.7)$ & 0.31 \\
\hline
\end{tabular}

Table 4 cross-classifies IS-OS status at baseline and at month 12 and presents mean baseline to month 12 change in CPT and in VA. Decrease in CPT was greater in eyes with absent IS-OS junction at baseline than in those with abnormal IS-OS junction. Change in VA appeared to show a trend with change in IS-OS status. In 16 eyes that remained with absent IS-OS status at 12 months, the mean VA decreased by 10.4 letters, in 6 eyes with improvement in IS-OS status from absent to abnormal mean VA remained essentially unchanged ( -0.8 letters), and in 8 eyes with improvement from absent to normal IS-OS junction mean VA improved by 8.9 letters. Results were similar for the 12 eyes with abnormal IS-OS junction at baseline, but not nominally significant, perhaps because of small numbers.

Photoreceptor length was ungradable in 27 (64\%) eyes. Visibility of OPL was a requirement for this measurement and this layer was disrupted in most eyes because of intraretinal fluid. Although the numbers were small, there was no significant correlation between photoreceptor length and IS-OS status at both baseline and month 12 .

\section{Discussion}

In this cohort of eyes with macular oedema because of RVO from the SCORE Study, IS-OS status evaluated with TD OCT scans showed a significant association between change in IS-OS status and change in VA from baseline to 1 year. Independent of baseline category, IS-OS status returning to normal was associated with improvement in VA, and IS-OS in the absent category was associated with the loss of VA. Although there seemed to be a significant relationship between change in IS-OS status and change in VA, the range of VA letter score was wide. Interestingly, eyes that remained in the absent IS-OS category at month 12 did not remain stable in their VA, but instead showed a mean loss in VA letter score.

At baseline, IS-OS status was significantly associated with OCT-measured CPT, but not with VA and photoreceptor length. There were no cases with normal
IS-OS status at baseline, indicating that outer retinal status is often affected in cases where there is VA impairment associated with macular oedema due to RVO. At month 12, IS-OS status was significantly associated with VA and OCT-measured CPT, but not with photoreceptor length. The association between thick retina and absent IS-OS junction at both baseline and follow-up visits raises the possibility that thickened retina confounds IS-OS assessment. Despite this potential confounder, change in IS-OS status between baseline and 12 months had a stronger correlation with change in VA than change in central retinal thickness (Table 3).

Image quality issues were common and may have confounded assessment, particularly at baseline. The IS-OS junction is not a true tissue interface and possibly represents a change in refractive index between the inner and outer segments of the photoreceptors. ${ }^{27}$ It is difficult to distinguish loss of IS-OS junction due to photoreceptor damage from absence of IS-OS junction due to various imaging artifacts. Overlying cysts haemorrhage and hard exudates may attenuate the reflectivity of deeper retinal layers, thereby decreasing the sensitivity of the classification of outer retina morphology. At baseline, the IS-OS status may have been misclassified as absent, not because of a structural abnormality but because of poor imaging of the layer. By month 12, the cysts, subretinal fluid and intraretinal haemorrhage had resolved in most eyes and, thus, were not an impediment to imaging the outer retina. At this time, we are unable to distinguish between absence of the IS-OS due to obscuration by artifact and absence of the layer due to pathology.

Recent studies have found correlations between IS-OS status and VA in various diseases in TD OCT and more recently SD OCT. ${ }^{12-14,23,24,28-33}$ Recent publications on using SD OCT further support the view that outer retinal integrity is an important factor for visual outcome in eyes with RVO. ${ }^{32,33}$ However, there is lack of long-term follow-up in a prospective standardized fashion using SD OCT. The current ancillary study was performed to 
assess the relationship between change in IS-OS and change in VA status in a randomized clinical trial with a standardized visit schedule, imaging protocol, and VA measurements. Although high resolution TD OCT scans (512 A scans/B scans) with resolution similar to some SD OCT macular volume scan ('cube') protocols were used for evaluation, only two B scans were available in the cross hair protocol of the TD OCT in contrast with SD OCT where many more B scans are available for assessment of IS-OS status. Agreement between Topcon 3D OCT scans and Stratus cross hairs for status of the IS-OS junction in the central subfield using the three step scale was $80.6 \%, \kappa 0.7$ (unpublished data). Thus, the results from this study using TD OCT are likely applicable to SD OCT.

Recent literature has focused on outer segment length as an important predictor of VA. ${ }^{8,9}$ We did not analyze outer segment length in this sample due to the lack of tools for the required minute measurements in the manufacturer's software. Photoreceptor length has also been found to be correlated with VA in macular oedema from RVO $(r=-0.571)^{14}$ but in our experience is difficult to define in eyes with macular oedema due to RVO.

This SCORE ancillary study suggests that IS-OS status is positively correlated with VA and is a promising assessment that warrants further investigation. However, this variable is susceptible to artifacts and must be interpreted with caution. A comprehensive outer retinal morphology assessment including status of IS-OS, photoreceptor length and outer segment length measurements may be a useful endpoint in clinical trials.

\section{Summary}

\section{What was known before}

- OCT central retinal thickness and VA correlate moderately outer retinal changes correlate with VA in retrospective and short-term prospective studies.

\section{What this study adds}

- Twelve month follow-up on standardized OCT data with concurrent VA shows outer retinal morphology correlates with concurrent VA and change in outer retinal morphology correlates with change in VA.

\section{Conflict of interest}

The authors declare no conflict of interest.

\section{References}

1 Ip MS, Oden NL, Scott IU, Vanveldhuisen PC, Blodi BA, Figueroa $\mathrm{M}$ et al. SCORE Study Report 3 Study Design and Baseline Characteristics. Ophthalmology 2009; 116(9): 1770-1777.

2 DRCRnet. A Randomized Trial comparing intravitreal triamcinolone acetonide and focal/grid photocoagulation for diabetic macular edema. Ophthalmology 2008; 115(9): 1447-1459.

3 Elman MJ, Aiello LP, Beck RW, Bressler NM, Bressler SB, Edwards AR et al. Randomized trial evaluating ranibizumab plus prompt or deferred laser or triamcinolone plus prompt laser for diabetic macular edema. Ophthalmology 2010; 117(6): 1064-1077.

4 Fung AE, Lalwani GA, Rosenfeld PJ, Dubovy SR, Michels S, Feuer WJ et al. An optical coherence tomography-guided, variable dosing regimen with intravitreal ranibizumab (Lucentis) for neovascular age-related macular degeneration. Am J Ophthalmol 2007; 143(4): 566-583.

5 Altaweel MM, Hubbard L, Gagnon R, Eyetech Study Group. The utility of optical coherence tomography in eligibility determination for clinical trials of macular disease therapy. invest. Invest Ophthalmol Vis Sci 2005; 46: E-Abstract 1577.

6 Scott IU, VanVeldhuisen PC, Oden NL, Ip MS, Blodi BA, Jumper JM et al. SCORE Study Report 1: Baseline associations between central retinal thickness and visual acuity in patients with retinal vein occlusion. Ophthalmology 2009; 116(3): 504-512.

7 Browning DJ, Glassman AR, Aiello LP, Beck RW, Brown DM, Fong DS et al. Relationship between optical coherence tomography-measured central retinal thickness and visual acuity in diabetic macular edema. Ophthalmology 2007; 114(3): 525-536.

8 Forooghian F, Stetson PF, Meyer SA, Chew EY, Wong WT, Cukras C et al. Relationship between photoreceptor outer segment length and visual acuity in diabetic macular edema. Retina 2010; 30(1): 63-70.

9 Alasil T, Keane PA, Updike JF, Dustin L, Ouyang Y, Walsh AC et al. Relationship between optical coherence tomography retinal parameters and visual acuity in diabetic macular edema. Ophthalmology 2010; 117(12): 2379-2386.

10 Keane PA, Liakopoulos S, Chang KT, Wang M, Dustin L, Walsh AC et al. Relationship between optical coherence tomography retinal parameters and visual acuity in neovascular age-related macular degeneration. Ophthalmology 2008; 115(12): 2206-2214.

11 Suh MH, Seo JM, Park KH, Yu HG. Associations between macular findings by optical coherence tomography and visual outcomes after epiretinal membrane removal. Am J Ophthalmol 2009; 147(3): 473-480.

12 Ota M, Tsujikawa A, Kita M, Miyamoto K, Sakamoto A, Yamaike $\mathrm{N}$ et al. Integrity of foveal photoreceptor layer in central retinal vein occlusion. Retina 2008; 28(10): 1502-1508.

13 Hayashi H, Yamashiro K, Tsujikawa A, Ota M, Otani A, Yoshimura N. Association between foveal photoreceptor integrity and visual outcome in neovascular age-related macular degeneration. Am J Ophthalmol 2009; 148: 83-90.

14 Ota M, Tsujikawa A, Murakami T, Yamaike N, Sakamoto A, Kotera Y et al. Foveal photoreceptor layer in eyes with persistent cystoid macular edema associated with branch retinal vein occlusion. Am J Ophthalmol 2008; 145(2): 273-280.

15 Chan A, Duker JS, Ishikawa H, Ko TH, Schuman JS, Fujimoto JG. Quantification of photoreceptor layer thickness in normal eyes using optical coherence tomography. Retina 2006; 26(6): 655-660

16 Szkulmowski M, Wojtkowski M, Sikorski B, Bajraszewski T, Srinivasan VJ, Szkulmowska A et al. Analysis of posterior retinal layers in spectral optical coherence tomography images of the normal retina and retinal pathologies. J Biomed Opt 2007; 12(4): 041207. 
17 Garvin MK, Abramoff MD, Wu X, Russell SR, Burns TL, Sonka M. Automated 3-D intraretinal layer segmentation of macular spectral-domain optical coherence tomography images. IEEE Trans Med Imaging 2009; 28(9): 1436-1447.

18 Srinivasan VJ, Monson BK, Wojtkowski M, Bilonick RA, Gorczynska I, Chen $\mathrm{R}$ et al. Characterization of outer retinal morphology with high-speed, ultrahigh-resolution optical coherence tomography. Invest Ophthalmol Vis Sci 2008; 49(4): 1571-1579.

19 Bagci AM, Shahidi M, Ansari R, Blair M, Blair NP, Zelkha R. Thickness profiles of retinal layers by optical coherence tomography image segmentation. Am J Ophthalmol 2008; 146(5): 679-687.

20 Wojtkowski M, Sikorski BL, Gorczynska I, Gora M, Szkulmowski M, Bukowska D et al. Comparison of reflectivity maps and outer retinal topography in retinal disease by 3-D Fourier domain optical coherence tomography. Opt Express 2009; 17(5): 4189-4207.

21 Spaide RF, Curcio CA. Anatomical correlates to the bands seen in the outer retina by optical coherence tomography: literature review and model. Retina 2011; 31(8): 1609-1619.

22 Sadda SR, Wu Z, Walsh AC, Richine L, Dougall J, Cortez R et al. Errors in retinal thickness measurements obtained by optical coherence tomography. Ophthalmology 2006; 113(2): 285-293.

23 Oster SF, Mojana F, Brar M, Yuson RM, Cheng L, Freeman WR. Disruption of the photoreceptor inner segment/outer segment layer on spectral domain-optical coherence tomography is a predictor of poor visual acuity in patients with epiretinal membranes. Retina 2010; 30(5): 713-718.

24 Maheshwary AS, Oster SF, Yuson RM, Cheng L, Mojana F, Freeman WR. The association between percent disruption of the photoreceptor inner segment-outer segment junction and visual acuity in diabetic macular edema. Am J Ophthalmol 2010; 150(1): 63-67.

25 Ip MS, Scott IU, VanVeldhuisen PC, Oden NL, Blodi BA, Fisher $\mathrm{M}$ et al. A randomized trial comparing the efficacy and safety of intravitreal triamcinolone with observation to treat vision loss associated with macular edema secondary to central retinal vein occlusion: the Standard Care vs Corticosteroid for Retinal Vein Occlusion (SCORE) study report 5. Arch Ophthalmol 2009; 127(9): 1101-1114.

26 Scott IU, Ip MS, VanVeldhuisen PC, Oden NL, Blodi BA, Fisher $\mathrm{M}$ et al. SCORE Study Report 6 - A randomized trial comparing the efficacy and safety of intravitreal triamcinolone with standard care to treat vision loss associated with macular Edema secondary to branch retinal vein occlusion: the Standard Care vs Corticosteroid for Retinal Vein Occlusion (SCORE) study report 6. Arch Ophthalmol 2009; 127(9): 1115-1128.

27 Sherman J, Yannuzzi L, Madonna R, Nath S, Bababekova Y. Photoreceptor Integrity Line - As Revealed by Spectral Domain OCT. E-Book; 2009, p 1-224.

28 Spaide RF, Koizumi H, Freund KB. Photoreceptor outer segment abnormalities as a cause of blind spot enlargement in acute zonal occult outer retinopathy-complex diseases. Am J Ophthalmol 2008; 146(1): 111-120.

29 Mitamura Y, Aizawa S, Baba T, Hagiwara A, Yamamoto S. Correlation between retinal sensitivity and photoreceptor inner/outer segment junction in patients with retinitis pigmentosa. Br J Ophthalmol 2009; 93(1): 126-127.

30 Mitamura Y, Hirano K, Baba T, Yamamoto S. Correlation of visual recovery with presence of photoreceptor inner/outer segment junction in optical coherence images after epiretinal membrane surgery. Br J Ophthalmol 2009; 93(2): 171-175.

31 Aizawa S, Mitamura Y, Baba T, Hagiwara A, Ogata K, Yamamoto S. Correlation between visual function and photoreceptor inner/outer segment junction in patients with retinitis pigmentosa. Eye 2008; 23(2): 304-308.

32 Shin HJ, Chung H, Kim HC. Association between integrity of foveal photoreceptor layer and visual outcome in retinal vein occlusion. Acta Ophthalmologica 2011; 89(1): e35-e40.

33 Wolf-Schnurrbusch UE, Ghanem R, Rothenbuehler SP, Enzmann V, Framme C, Wolf S. Predictors of short-term visual outcome after Anti-VEGF therapy of macular oedema due to central retinal vein occlusion. Inves Ophthalmol Vis Sci 2011; 52(6): 3334-3337. 